\title{
A STOP CODON POLYMORPHISM OF TOLL-LIKE RECEPTOR 5 IS ASSOCIATED WITH A STABLE COURSE OF CHRONIC OBSTRUCTIVE LUNG DISEASE
}

\author{
Stefan Pabst*, Vildan Yenice*, Martina Lennarz*, Georg Baumgarten*, Pascal Knuefermann", Bettina Hecht \\ Adrian Gillissen ", Hans Vetter* and Christian Grohe* \\ *Medical Policlinic, University Hospital, Bonn, Germany \\ \#Dept of Anesthesiology, University Hospital, Bonn, Germany \\ 'Dept of Pneumology, St. George Medical Center, Leipzig, Germany
}

WINNING ABSTRACT: The etiology of chronic obstructive lung disease (COPD) is unclear. It is supposed to be the product of an exogenous antigenic stimulus, such as tobacco smoke, and an endogenous genetic susceptibility. Toll-like receptors (TLR) are signal molecules, essential for the cellular response to bacterial cell wall components. Lipopolysaccharide (LPS) binds to TLR4 and two different polymorphisms for the TLR4 gene (Asp299Gly and Thr399lle) have recently been described. TLR5 is the receptor for flagellin, aconstituent of Gram-positive and -negative bacterial flagella. A functional relevant TLR5 polymorphism (TLR5392STOP) has already been identified. The coactivation of both TLR4 and 5 seems to play an important role in the mediation of host-defense mechanisms. We genotyped 138 Caucasian patients with COPD and 135 healthy controls for the TLR5 polymorphism TLR5392STOP respectively for Asp299Gly and Thr399lle polymorphisms in theTLR4gene. Among COPD patients the prevalence for the TLR5 mutant allele was $9.42 \%(26 / 276)$. The prevalence for each Asp299Gly and Thr399lle mutant allele was $4.71 \%(13 / 276)$. In the control group the TLR5 mutation prevalence was $5.19 \%(14 / 270)(P=0.138)$, the prevalence of each TLR4 polymorphism was $2.96 \%(8 / 270)(p=0.279)$. In the subgroup of 54 patients with a stable course of COPD, defined as less than three hospitalizations over the last three years due to COPD, we found a significant association with the TLR5392STOP gene polymorphism $(P=0.026)$. These data suggest that the TLR5392STOP polymorphism is associated with a stable course of COPD, whereas TLR4 polymorphisms have no impact neither on the onset nor on the course of COPD.

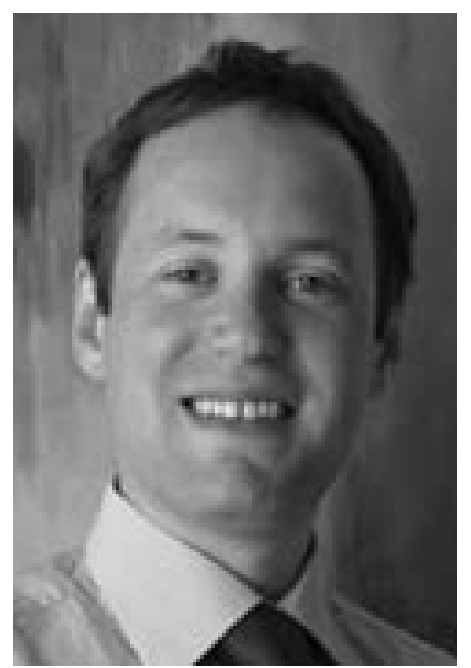

Stefan Pabst

Medizinische Klinik II, Department of Pneumology, University Hospital of Bonn, Bonn, Germany

\section{MY JOB AND THE UNIT IN WHICH I WORK}

I work at the Dept of Pneumology at the University Hospital of Bonn, Bonn, Germany. Besides my work as the ward physician on "Station Rühle", I also work in the outpatient unit. We offer the whole spectrum of clinical pneumology, including flexible and rigid bronchoscopy with endobronchial stenting. The head of the department is Prof. Christian Grohé, whom I would like to thank for his support and effort in the clinical studies over the last few years.

\section{MY WINNING POSTER AS PART OF MY RESEARCH}

My scientific interest is the investigation of the aetiology of chronic obstructive pulmonary disease (COPD), sarcoidosis and obstructive sleep apnoea. We know that for all these diseases, there is a genetic background that is probably polygenetically inherited. Therefore, we (the Bonn study group) are attempting to collect all patients with these diseases in clinical studies to evaluate exogenous risk factors. We also examine DNA from blood samples to perform case-control studies for specifying the individual genetic pattern. Besides these cohorts, we have recruited a group of healthy volunteers, predominantly from pre-employment examinations in our clinic. In the laboratory, we use restriction fragment length polymorphism analysis, as well as real-time PCR.

\section{MY RESEARCH AS PART OF MY WORKING GROUP/ RESEARCH TEAM}

Progressive airflow limitation due to chronic obstructive bronchitis and emphysema is the main characteristic of COPD. At the moment, COPD ranks fourth as a global cause of death and shows a worldwide increase both in morbidity and mortality. This development has provoked rising interest in the clarification of the pathogenic mechanisms underlying this common disease, in order to develop appropriate and effective therapeutic interventions. Environmental and genetic determinants, as well as their interactions, influence COPD susceptibility. The most significant environmental contributor is tobacco smoke. However, not all smokers develop COPD, which leads us to the conclusion that other factors contribute to the onset of COPD. Moreover, in most cases, the course of COPD is not determined by the number of cigarettes smoked or the actual smoking habits. As it is unclear which 
determinants cause the onset or modify the course of COPD, it is the aim of our studies to find out in which way genetic factors are at play.

Meanwhile, different candidate genes besides $\alpha_{1}$-antitrypsin deficiency have been found that may be a cause of COPD. Genomic approaches have investigated multiple candidate genes, but with inconsistent results. One of the most recent and best examples for this hypothesis is the widely regarded study by DEMEO et al. [1], which showed an association between the SERPINE2 gene and COPD. However, the excellent results of the study by DEMEO et al. [1] could not be repeated in an independent sample [2]. This shows that the aetiology of COPD is much more complex and polygenic, as previously expected. A large problem in the search for candidate genes by means of case-control studies is, of course, the impact of population stratification, as ethnic and regional dependence influences the results of a case-control study. Therefore, in our opinion, the quality and the results of this type of study are highly dependent on the selected patient group, as well as the control group. Thus, the Bonn study group, which consists of patients and healthy volunteers selected only from the surroundings of the city of Bonn is sorted into those patients and volunteers with a different origin, especially a different ethnic background, by use of a questionnaire. However, as can be learnt from the examples mentioned previously [1, 2], even when the greatest of care has been taken in the assortment of the sample, different findings may still result.

A second problem encountered when collecting and observing large sample groups is the phenotypic heterogeneity. As in many other human diseases, complex inherited disorders show a progressive distribution from the "normal" phenotype towards the "diseased" phenotype. In our opinion, an exact characterisation of the phenotype is of high importance for the validity of the results. In terms of COPD, this could mean that stratification could be carried out by means of forced expiratory volume in one second/Global Initiative for Chronic Obstructive Lung Disease (GOLD) classification, by clinical stratification (pink puffer/blue bloater), or by exercise capacity. It is possible that this list could be extended even further. In our group, we have examined candidate genes that alter the innate and acquired immune response, which are most likely to lead to exacerbations of COPD and which subsequently leads to hospital admissions. As these exacerbations are often life-threatening, expensive and result in a loss of life quality, we decided to divide our COPD group into two groups: one subgroup with frequent exacerbations and another subgroup with a stable disease. Of course, we cannot be sure if this is the correct phenotyping so, during the course of our study, we must observe if this classification proves to be the most appropriate option. However, we think that without exact classification of the phenotype and subsequent division into subgroups, it will be very difficult to reproduce results in different samples. Only by careful investigation will it be possible to determine the complex genetic patterns that influence the course of a non-Mendelian-inherited disease, such as COPD.

In conclusion, there are at least two routes in case-control studies that may lead to appropriate results, as follows: 1) amplification of the sample size, either in one single centre or as a multicentre study; and 2) isolation of the exact characterisation of the phenotype that may lead, even in smaller sample sizes, to the same results. In the Bonn study group, we try to follow both of these methods by means of precise phenotyping and continuous enlargement of the cases, as well as of the healthy controls.

With regard to sarcoidosis, in March 2006 we established the Bonn sarcoidosis network [3]. The aim of the network is the optimised treatment of patients with sarcoidosis, because we have realised that it often takes a long time from the appearance of the first symptoms to the right diagnosis and treatment. We are also able to recruit many patients from the surrounding areas of Bonn for our case-control studies. Before the start of the network, we had nearly 200 patients with sarcoidosis in our database. Following the establishment of the network, in just 6 months, we were able to add 100 more patients. This project is supported by institutional grants (from BONFOR; the BONn FORschung, an institutional programme for young scientists of the University Hospital of Bonn) for the next 2 yrs.

As in COPD, the course of sarcoidosis may be altered genetically. We see patients with an acute course, mostly as Loefgren's syndrome, which is self-limiting and does not require treatment with corticosteroids. There are also patients who develop a chronic (recurrent) course of sarcoidosis, sometimes with a fatal outcome. Although the phenotype of most forms of sarcoidosis appears to be similar, we propose that what we see are different phenotypes that are genetically

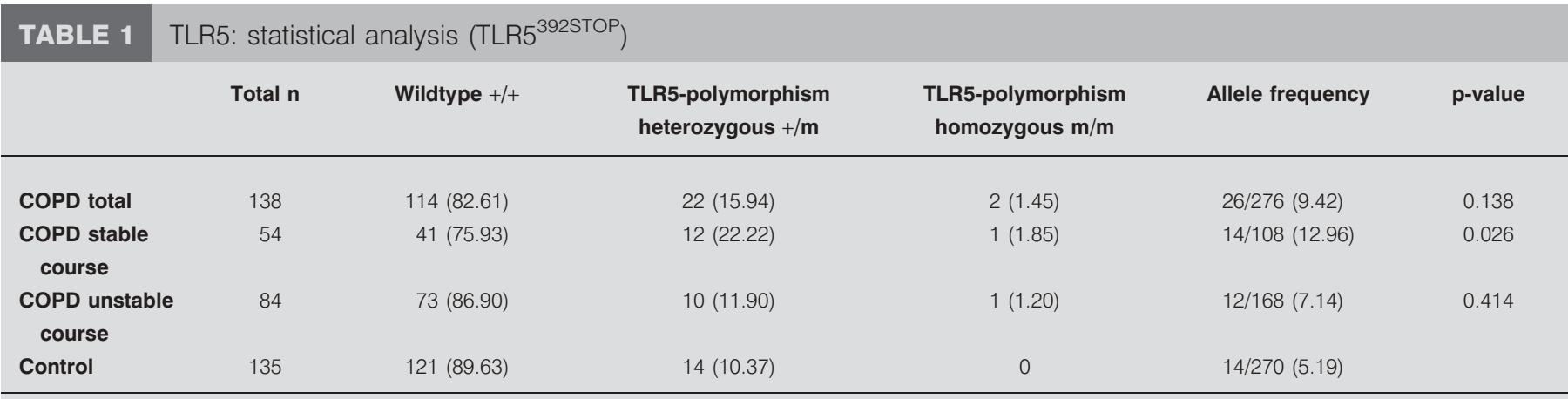

Data are presented as $n(\%)$, unless otherwise stated. TLR: Toll-like receptor 
TABLE 2 TLR4: statistical analysis (Asp299Gly/Thr399lle)

\begin{tabular}{|c|c|c|c|c|}
\hline Total $\mathbf{n}$ & Wildtype $+/+$ & $\begin{array}{l}\text { TLR4-polymorphism } \\
\text { heterozygous }+/ m\end{array}$ & $\begin{array}{c}\text { TLR4- } \\
\text { polymorphism } \\
\text { homozygous } \mathrm{m} / \mathrm{m}\end{array}$ & Allele frequency \\
\hline
\end{tabular}

\begin{tabular}{lccccc} 
COPD total & 138 & $124(89.86)$ & $14(10.14)$ & 0 & $14 / 276(5.07)$ \\
COPD stable course & 54 & $47(87.04)$ & $7(12.96)$ & 0 & $7 / 108(6.48)$ \\
COPD unstable course & 84 & $77(91.67)$ & $7(8.33)$ & 0 & $7 / 168(4.17)$ \\
Control & 135 & $128(94.87)$ & $7(5.13)$ & 0 & 0.136 \\
\hline
\end{tabular}

Data are presented as $n(\%)$, unless otherwise stated. TLR: Toll-like receptor.

a) 150

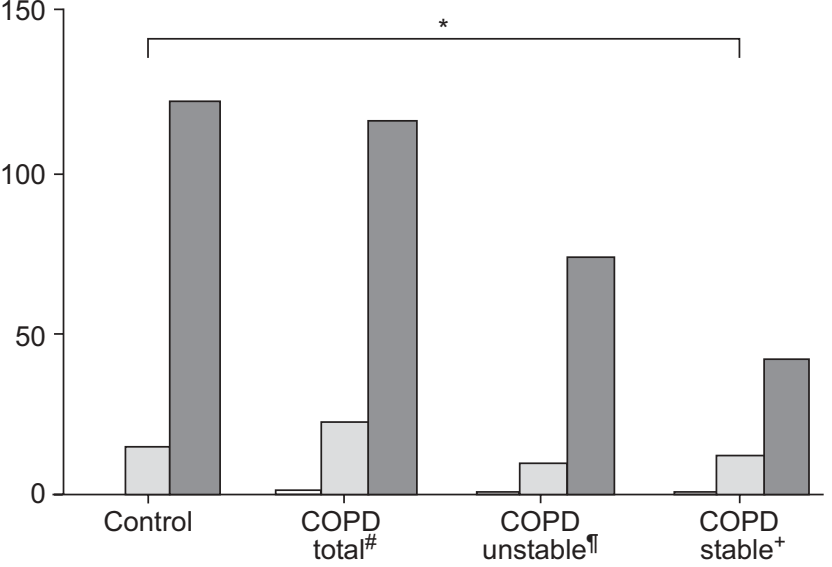

b) 150

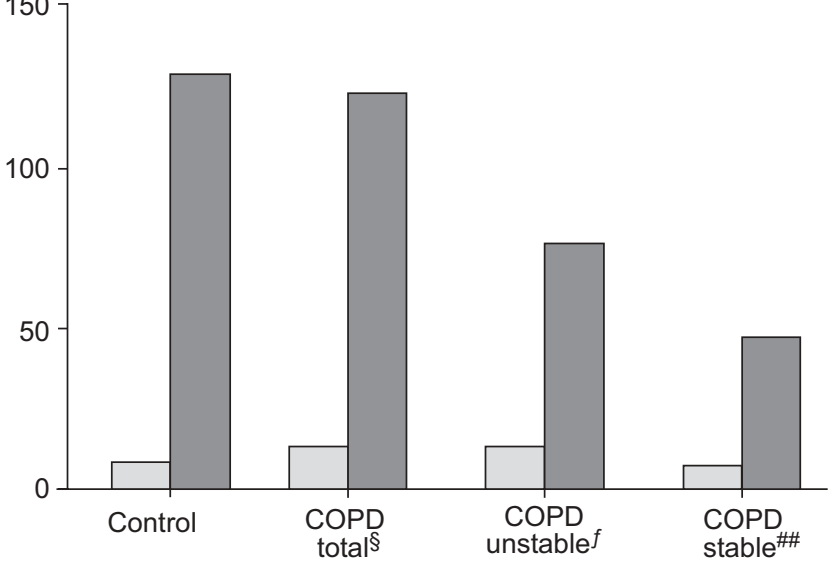

FIGURE 1. Statistical analysis. Course of chronic obstructive pulmonary disease (COPD) in a) Toll-like receptor (TLR)5 and b) TLR4. a) $\square$ : C1174T homozygous; $\square$ : C1174T heterozygous; $\square$ : wildtype homozygous. b) $\square$ : Asp299Gly/Thr399lle heterozygous; $\square$ : wildtype homozygous. *: $p<0.05$; ${ }^{\#}: p=0.138 ;{ }^{\natural}: p=0.414 ;^{+}: p=0.026 ;{ }^{s}: p=0.124 ;{ }^{f}: p=0.226{ }^{\# \#}: p=0.136$.

determined. In our cohort, we found that a Toll-like receptor 4 polymorphism is associated with a chronic course of sarcoidosis [4]. Moreover, we were able to repeat the results of VALENTONYTE et al. [5], who showed that sarcoidosis is associated with a polymorphism in butyrophilin-like-2 gene, which alters T-cell activation. However, in addition to that work, we were also able to show that the association is observed only in the chronic forms of sarcoidosis [6].

As a new project, we started the recruitment of patients with sleep apnoea. In our hospital ward, we examine six patients a week in our sleep laboratory. In addition to this, we see $\sim 40$ patients per week for follow-up in our outpatient unit. We hope that in 1 yr we will have recruited $>1,500$ patients with sleep apnoea, in order to perform case-control studies in this cohort.

\section{THE IMPACT OF MY WORK ON CLINICAL OR RESEARCH PRACTICE}

In my opinion, our clinical research with performance of casecontrol studies will allow us to gain a better understanding of many diseases in a reasonable amount of time. I hope that in a few years we will be able to make a much more exact diagnosis and treat our patients according to the specific phenotype of disease. In oncology, the analysis of receptor expression has already found its way into clinical practice and I anticipate that this will also happen in many other diseases.

\section{REFERENCES}

1 DeMeo DL, Mariani TJ, Lange C, et al. The SERPINE2 gene is associated with chronic obstructive pulmonary disease. Am J Hum Genet 2006; 78: 253-264.

2 Chappell S, Daly L, Morgan K, et al. The SERPINE2 gene and chronic obstructive pulmonary disease. Am J Hum Genet 2006; 79: 184-187.

3 Sarkoidose Netzwerk Bonn. www.sarkoidose-bonn.de. Date last updated: February 27, 2007. Date last accessed: April 2, 2007.

4 Pabst S, Baumgarten G, Stremmel A, et al. Toll-like receptor (TLR) 4 Asp299Gly and Thr399Ile polymorphisms are associated with a chronic progressive course of pulmonary sarcoidosis. Clin Exp Immunol 2006; 143: 420-426.

5 Valentonyte R, Hampe J, Huse K, et al. Sarcoidosis is associated with a truncating splice site mutation in BTNL2. Nat Genet 2005; 37: 357-364.

6 Li Y, Wollnik B, Pabst S, et al. A BTNL2 gene variant confers to sarcoidosis susceptibility by an increased risk towards the chronic form of the disease. Thorax 2006; 61: 273-274. 